\title{
Sobrecarga de fluidos en pacientes hospitalizados en una institución de segundo nivel en México
}

\author{
Fluid overload in hospitalized patients at a second-level institution in Mexico
}

Daniela F Sánchez Torres,* Ángel Tlacuílo Morales, ${ }^{\ddagger}$ Abraham Arias González§

\begin{abstract}
RESUMEN
Introducción: La sobrecarga de fluidos es definida como un balance positivo acumulado de líquidos que representa un mecanismo de descompensación aguda asociada con el agravamiento de síntomas, la hospitalización tardía o incluso la muerte. El objetivo es determinar la influencia de sobrecarga de fluidos en la evolución de los pacientes hospitalizados, así como conocer el servicio que tiene la mayor prevalencia de sobrecarga hídrica. Material y métodos: Se desarrolló un estudio de tipo transversal, comparativo y retrospectivo. La muestra fue de 387 pacientes hospitalizados, las comparaciones transversales entre grupos para variables cuantitativas se realizaron mediante análisis de varianza. Se aplicó la prueba de contrastes de Tukey, y análisis de correlación de Pearson. Resultados: Se encontró una prevalencia global de pacientes con sobrecarga de fluidos (> 10\%) del $7.75 \%(n=30)$. En las hojas de enfermería coincidió el registro de 250 pacientes, es decir, 66.6\% de los casos, en el restante (33.4\%) se encontraron diferencias en la cuantificación del balance hídrico. Hallando que no existe relación estadística significativa entre el índice de sobrecarga > 10\% y los días de estancia intrahospitalaria en cualquier servicio ( $r \leq 0.01 ; p=0.94$ ). Conclusiones: Se logró enfatizar que el personal de enfermería y equipo de salud involucrado en el manejo de fluidos electrolíticos del paciente hospitalizado es de gran importancia para su egreso, ya que es fundamental para evitar complicaciones derivadas de la sobrecarga de fluidos donde predominan fallas orgánicas, además de brindar un buen manejo hospitalario durante su estancia y disminuir costos en la atención de salud.
\end{abstract}

Palabras clave: Sobrecarga de líquidos, hospitalización, egreso paciente.

\begin{abstract}
Introduction: Fluid overload is defined as a positive accumulated balance of liquids, which represents a mechanism of acute decompensation associated with the worsening of symptoms, late hospitalization or even death. The objective is to determine the influence of fluid overload on the evolution of hospitalized patients, as well as to know the service that has the highest prevalence of water overload. Material and methods: A cross-sectional, comparative and retrospective study was developed. The sample was 387 hospitalized patients, cross-sectional comparisons between groups for quantitative variables were performed by analysis of variance. Tukey's contrast test and Pearson's correlation analysis were applied. Results: A global prevalence of patients with fluid overload (> 10\%) of $7.75 \%(n=30)$ was found. In the nursing sheets, the registry of 250 patients coincided, that is, $66.6 \%$ of the cases, in the remaining $(33.4 \%)$ differences were found in the quantification of the water balance. Finding that there is no significant statistical relationship between the overload index $>10 \%$ and the days of hospital stay in any service $(r$ $\leq 0.01 ; p=0.94$ ) Conclusions: It was emphasized that the nursing staff and health team involved In the management of electrolytic fluids of the hospitalized patient it is of great importance for their discharge, since it is essential to avoid complications derived from fluid overload where organic failures predominate, in addition to providing good hospital management during their stay and reducing costs in the health care.
\end{abstract}

* Pasante de Licenciatura en Enfermería de la Universidad del Valle de México Campus Sur sede Coyoacán. Ciudad de México, México.

¥ Jefe de Urgencias en el Hospital General Tláhuac. Ciudad de México, México. ${ }^{\S}$ Director Nacional de Enfermería y Campos Clínicos de la Universidad del Valle de México. Ciudad de México, México.

Correspondencia: DFST, dfst_1995@hotmail.com Conflicto de intereses: Para la elaboración del presente estudio, no se declararon conflicto de intereses por parte de ninguno de los involucrados. Citar como: Sánchez Torres DF, Tlacuílo Morales A, Arias González A. Sobrecarga de fluidos en pacientes hospitalizados en una institución de segundo nivel en México. Rev CONAMED 2020; 25(3): 107-114. https://dx.doi. org/10.35366/95982 Financiamiento: Ninguno.

Recibido: 05/03/2020. Aceptado: 14/08/2020

Keywords: Fluid overload, hospitalization, patient discharge. 


\section{INTRODUCCIÓN}

La sobrecarga de fluidos es definida como un balance positivo acumulado de líquidos que representa un mecanismo de descompensación aguda y se asocia con el agravamiento de síntomas, la hospitalización tardía o incluso la muerte.' Los pacientes hospitalizados requieren de la administración de fluidos intravenosos (IV) y electrolitos por uno o más de los siguientes motivos: 1) resucitación hídrica, 2) mantenimiento de rutina, 3) reemplazo de líquidos y 4) redistribución de líquidos.²

Por lo tanto, el mal manejo de los fluidos en los pacientes hospitalizados es común, y puede generar consecuencias adversas para los pacientes, como excesiva morbilidad y mortalidad. Determinar el balance hídrico puede ser complejo y depende, en gran medida, de la fisiopatología subyacente; sin embargo, además del balance simple, los nuevos biomarcadores pueden ser útiles para la detección e identificación de riesgo de insuficiencia cardiaca (IC) descompensada aguda que puede permitir una pronta intervención, la cual conlleva mejores resultados clínicos. Reportes recientes han mostrado la importancia del balance hídrico tanto en adultos como en pacientes pediátricos con fracaso renal agudo (FRA). En general, un balance positivo augura mayor morbilidad y un mayor riesgo de peor resultado clínico. Así, el balance hídrico debe reconocerse como un biomarcador potencialmente modificable y determinante del resultado clínico en estos pacientes. ${ }^{2}$

La IC y el FRA son comunes, pero no se descartan otras patologías que pueden ser consideradas como de alto impacto y se asocian a gran consumo de recursos de salud, así como una morbimortalidad considerable. En este artículo, se proporciona una visión general de la acumulación de fluidos enfocada a los diferentes servicios de un hospital de segundo nivel, así como la importancia de la evaluación del balance hídrico en estos síndromesy correlación con los resultados por los profesionales de enfermería. ${ }^{2}$

La retención de líquidos intrahospitalaria puede llegar a ser de hasta 86\% en pacientes críticos y se ha asociado con una evolución desfavorable, propiciando dificultad a la deambulación y alteraciones cardiovasculares una vez egresados., ${ }^{3,4} \mathrm{El}$ balance hídrico positivo está asociado con un incremento de las tasas de mortalidad. ${ }^{5}$ Las principales complicaciones pueden ser IC y FRA que suelen ser comunes, pero no se descartan otras patologías que pueden ser consideradas como alto impacto y éstas pueden ser neurológicas, respiratorias, cardiacas, neuromusculares y hematológicas (Tabla 7).,6,7

Los fluidos cristaloides se distribuyen por el fluido extra celular (FEC) que tienen un efecto limitado y temporal sobre el volumen de plasma. Sin embargo, este tipo de conclusiones provienen de estudios realizados en sujetos euvolémicos sanos. Se ha observado que la retención intravascular de fluidos cristaloides es mayor en pacientes hipovolémicos que sugerido por este tipo de estudios, por lo cual existe incertidumbre si existe alguna ventaja de los líquidos coloides sobre cristaloides, en cuanto a la expansión. ${ }^{2}$

El reconocimiento y la evaluación de la sobrecarga de líquidos requiere un registro preciso de

\section{Tabla 1: Principales complicaciones.}

\begin{tabular}{ll}
\hline Órgano afectado & Complicaciones \\
\hline Cerebro & Delirio, déficit neurológico \\
Corazón & Alteración de la conducción, contractilidad deteriorada, disfunción diastólica \\
Pulmón & Intercambio de gases deteriorado, complianza reducida, aumento del trabajo respiratorio \\
Riñón & Aumento de la presión intersticial, lesión renal aguda, uremia, retención de líquidos \\
Hígado & Colestasis, hipertensión portal, síndrome hepatorrenal \\
Intestino y estómago & Mala absorción, íleo \\
Tejidos blandos & Mala cicatrización de heridas, infecciones, úlceras por presión \\
\hline
\end{tabular}




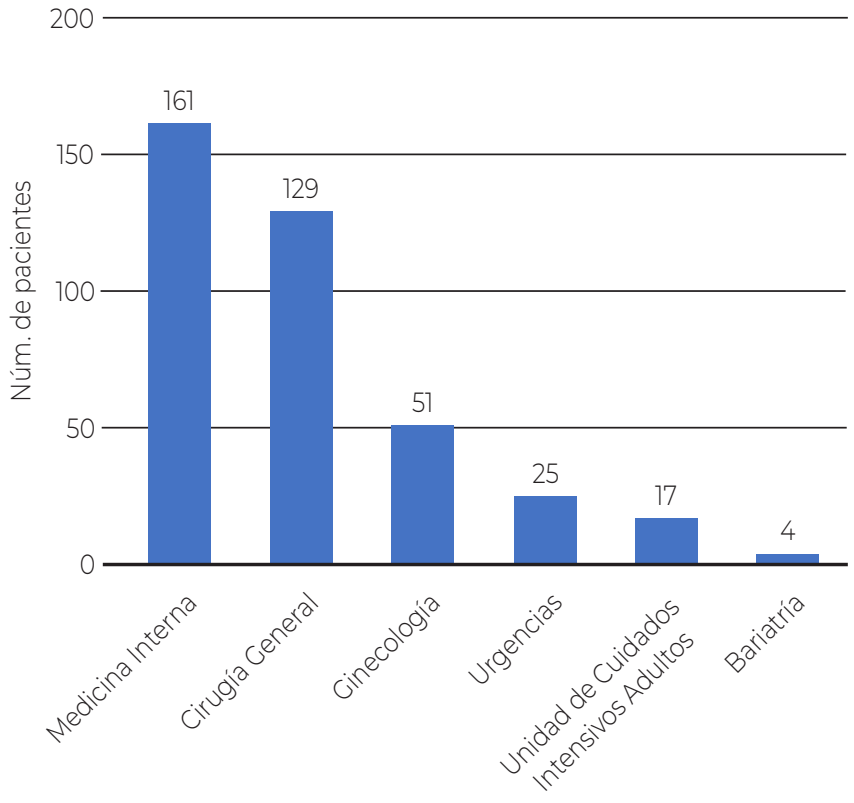

Figura 1: Número de pacientes por servicio.

ingresos y egresos; sin embargo, existe una amplia variación en la forma en que dicha información se registra, revisa y utiliza, algunas definiciones a utilizar son:2-6

1. Balance diario de fluidos: diferencia diaria en todos los ingresos y egresos, que con frecuencia no incluye pérdidas insensibles.

2. Balance acumulativo de líquidos: suma de cada día, balance de líquidos durante un periodo de tiempo.

3. Sobrecarga de líquidos: generalmente implica un grado de edema pulmonar o edema periférico.

4. Acumulación de fluidos: balance positivo de líquidos, con o sin sobrecarga de fluidos vinculada.

5. Porcentaje de sobrecarga de líquidos ajustada por el peso corporal (índice de sobrecarga): balance de líquido acumulado que se expresa como porcentaje. Un corte de $\geq 10 \%$ se ha asociado con un aumento de la mortalidad.

En cuanto al profesional de enfermería, quienes son los que participan en la prescripción y administración de líquidos por vía intravenosa, deben comprender diversos principios para que una terapia de líquidos resulte de una manera segura y eficaz, y para que a los pacientes no les genere consecuencias adversas como estancia prolongada y costos elevados para el sistema de salud.

El objetivo principal del presente estudio es determinar la influencia de la sobrecarga de fluidos en la evolución de los pacientes hospitalizados en un hospital de segundo nivel en México, así como conocer el servicio que tiene la mayor prevalencia de sobrecarga hídrica.

\section{MATERIAL Y MÉTODOS}

Se desarrolló un estudio de tipo transversal, comparativo y retrospectivo, en un hospital de segundo nivel en México. La muestra fue de 387 pacientes hospitalizados por el periodo comprendido del 2 de diciembre de 2018 al 15 de enero de 2019, en los servicios de Medicina Interna $(1,499)$, Cirugía General (1,217), Bariatría (316), Ginecología (3699). Se utilizaron como criterios de inclusión pacientes que estuvieron hospitalizados más de tres días, que en el expediente clínico se encontraran las medidas antropométricas (peso), registro de ingresos y egreso; se excluyeron expedientes que no contenían hoja de registro clínico de enfermería. Para la recolección de datos se utilizaron

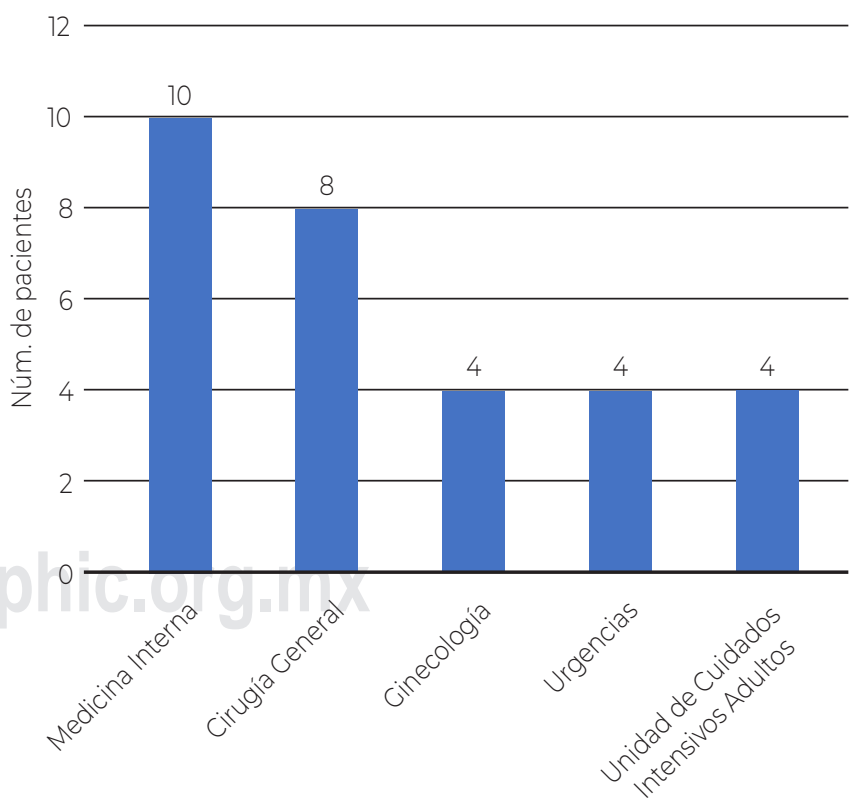

Figura 2: Número de pacientes con índice de sobrecarga > $10 \%$ por servicio. 


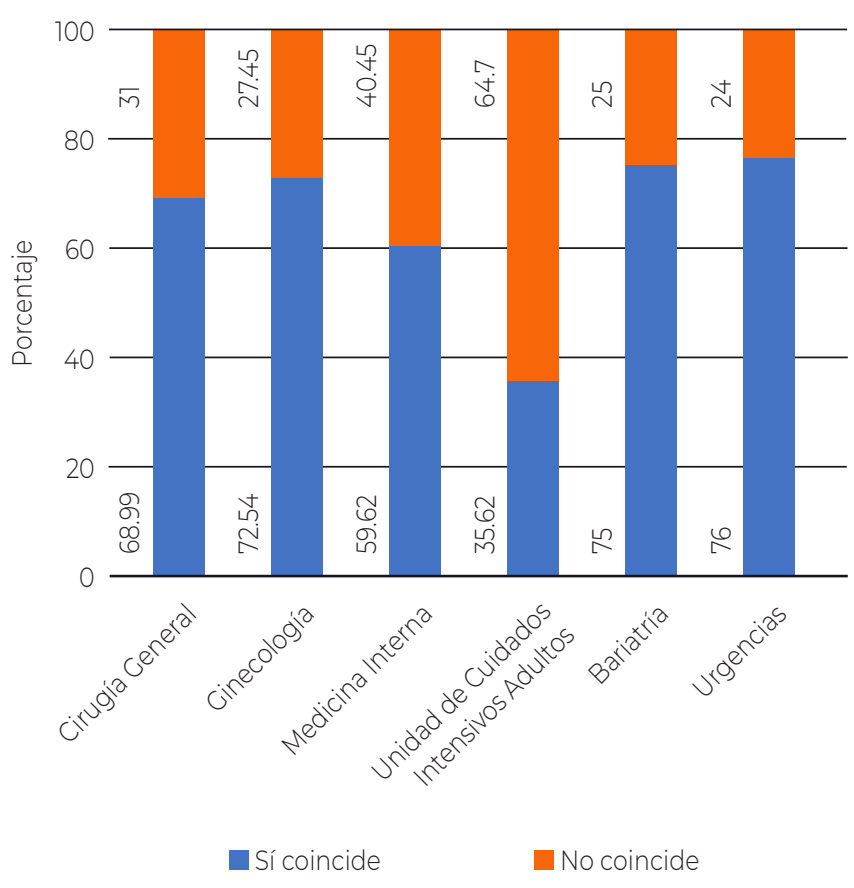

Figura 3: Porcentaje de no coincidencia de registro por servicio.

seis variables: sobrecarga de fluidos, índice de sobrecarga de fluidos, (IS) ingresos, egresos, peso y evolución clínica.

Las comparaciones transversales entre grupos para variables cuantitativas se realizaron mediante análisis de varianza (ANOVA). Se aplicó la prueba de contrastes de Tukey, y un análisis de correlación de Pearson.

\section{RESULTADOS}

Se analizaron un total de 387 expedientes, la prevalencia global de pacientes con sobrecarga de fluidos (índice > 10\%) fue del 7.75\% ( $n=30$ ). Las áreas que se tomaron en cuenta para la realización del presente estudio fueron aquéllos que cuentan con servicio de hospitalización, de esta forma se revisaron 161 expedientes de Medicina Interna (MI), 129 de Cirugía General (CG), 51 de Ginecología y Obstetricia (GyO), 25 de Urgencias Adultos (UA), 17 de la Unidad de Cuidados Intensivos de Adultos (UCIA) y cuatro expedientes de Cirugía Bariátrica (CB) como se muestra en la Figura 7. De los 161 expedientes revisados en el Servicio de Medicina Interna, se encontró un índice de sobrecarga (IS)
> 10\% en 10 de los expedientes revisados, lo que equivale al $2.58 \%$ del total de la muestra.

La moda en el diagnóstico asociado con sobrecarga fue el choque séptico con tres registros. En el caso de Cirugía General, ocho de 129 pacientes obtuvieron un IS > 10\%, esto corresponde a un 2.06\% de su población, la moda en el diagnóstico asociada con sobrecarga fue apendicectomía con dos casos y colecistectomía (dos casos). Se encontraron cuatro de 51 pacientes con IS > 10\% en el Servicio de GyO, haciendo $1.03 \%$ de los hospitalizados en esa área, el diagnóstico más común asociado con sobrecarga fue el puerperio fisiológico. Urgencias obtuvo IS > 10\% en cuatro de 25 pacientes, equivalente a 1.03\% de la población estudiada, su diagnóstico más frecuente asociado con sobrecarga fue la diabetes mellitus descompensada.

En la UCIA tuvo cuatro registros de 17 con IS mayor a 10\%, equivalente al 1.03\% de su total, no se encontró moda en el diagnóstico asociado con sobrecarga. En el Servicio de Cirugía Bariátrica a comparación de los demás, no se detectó ningún paciente con IS > 10\% (Figura 2). Otro aspecto evaluado fue la coincidencia del registro de los balances realizados en las hojas de enfermería; es

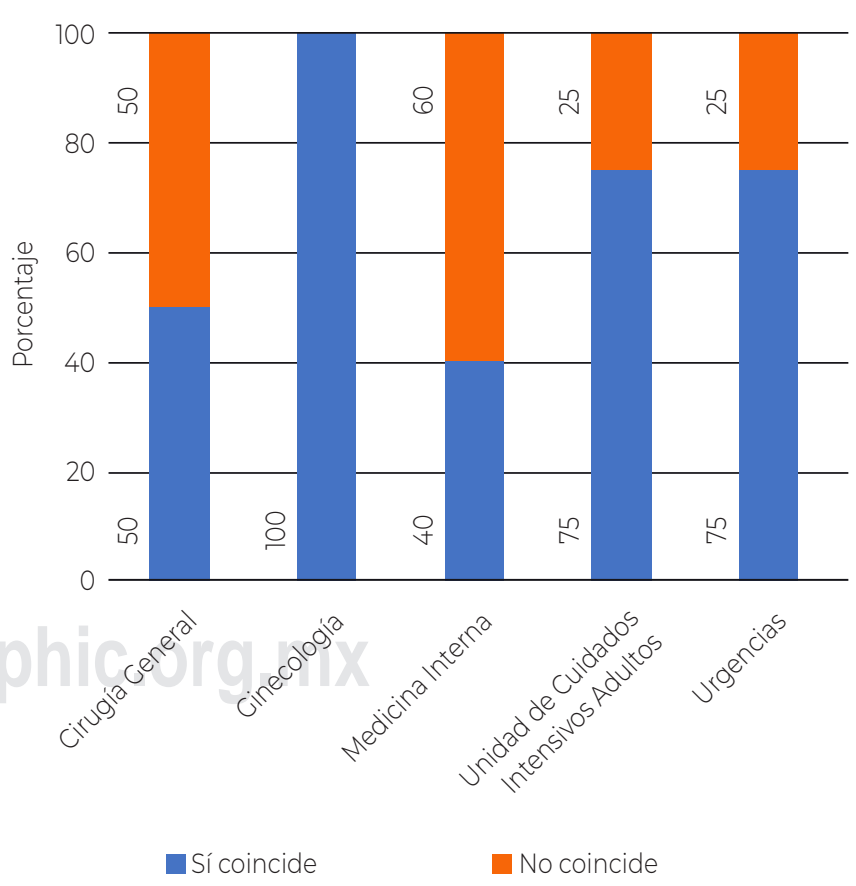

Figura 4: Porcentaje de pacientes con IS > 10\% que coincidieron en el registro. 


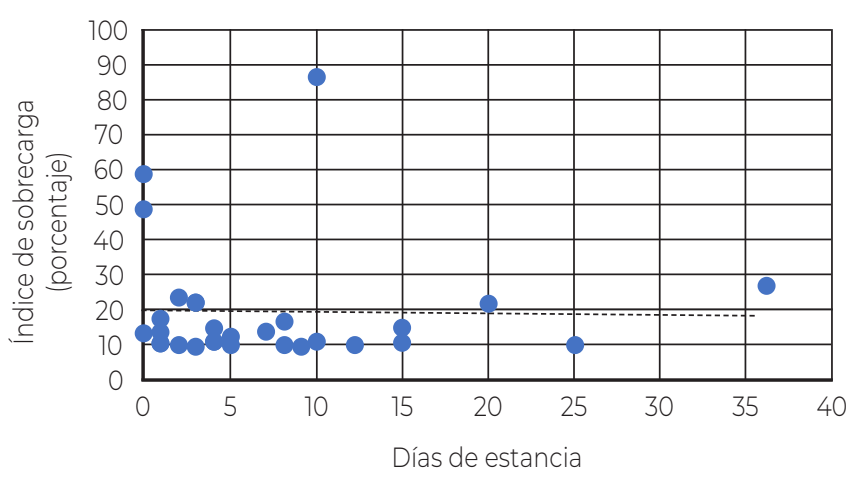

Figura 5: Relación entre días de estancia e índice de sobrecarga $>10 \%$.

decir, la cifra anotada en las mismas comparada con la cuantificación manual realizada por los investigadores del presente estudio. Del total de expedientes revisados, se encontró coincidencia de registro en 250 pacientes, es decir $66.6 \%$ de los casos, en el restante $33.4 \%$ se encontraron diferencias en la cuantificación del balance hídrico. Se realizó el análisis de frecuencias de dicho registro por servicio, encontrando que en MI 59.62\% de los expedientes no tenía un registro correcto.

En ese mismo servicio, del total de pacientes con IS > 10\%, el $40 \%$ no fue coincidente con la cuantificación manual realizada. Respecto de CG, se halló 31\% de no coincidencia en el total de sus registros, los pacientes con sobrecarga > 10\% de ese servicio tuvieron un error en la coincidencia de $50 \%$. En UCIA tuvo $64.77 \%$ de registros no coincidentes, para el caso de los hospitalizados con IS > 10\% el registro no coincidió en 25\%. El servicio de GyO obtuvo $27.47 \%$ de no coincidencia, en este caso el $100 \%$ de sus pacientes con sobrecarga presentaron concurrencia en el registro. Urgencias Adultos obtuvo la sincronía en el registro más alta con 66\% de registros concurrentes y $24 \%$ no, de todos los pacientes con IS > 10\% el 25\% no coincidieron en el registro de enfermería.

El área de CB obtuvo 100\% de sincronía de datos (Figuras 3 y 4). Uno de los objetivos principales de este estudio fue determinar la correlación existente entre el IS > 10\% y los días de estancia intrahospitalaria en todos los servicios; se encontró que no existe relación estadística significativa entre ambas variables ( $r \leq 0.01 ; p=0.94$ ), como se muestra en la Figura 5. De la misma forma, se realizó el análisis del IS > 10\% y los días de estancia intrahospitalaria por cada uno de los servicios estudiados. En el caso de GyO, URG y UCIA, el número de expedientes con sobrecarga mayor a 10\% fue de cuatro, por lo que no fue posible el análisis de correlación. Sin embargo, para el caso de CO se observa relación positiva, aunque no se encontró relevancia estadística ( $r=0.65 ; p=0.083)$ tal y como se muestra en la Figura 6.

El análisis del servicio de MI también arrojó una relación positiva entre días de estancia y sobrecarga, sin embargo, tampoco se obtuvo un valor estadísticamente significativo (Figura 7). Durante el estudio se observó que 123 expedientes de pacientes mostraron índice de sobrecarga negativo, se analizó de igual forma estos casos en cuanto a los días de estancia en este grupo; se encontró que no existe diferencia estadística entre dichas variables ( $r=0.02 ; p=0.82$ ), los resultados se muestran en la Figura 8. De igual manera, se realizó el mismo análisis para los expedientes con índice de

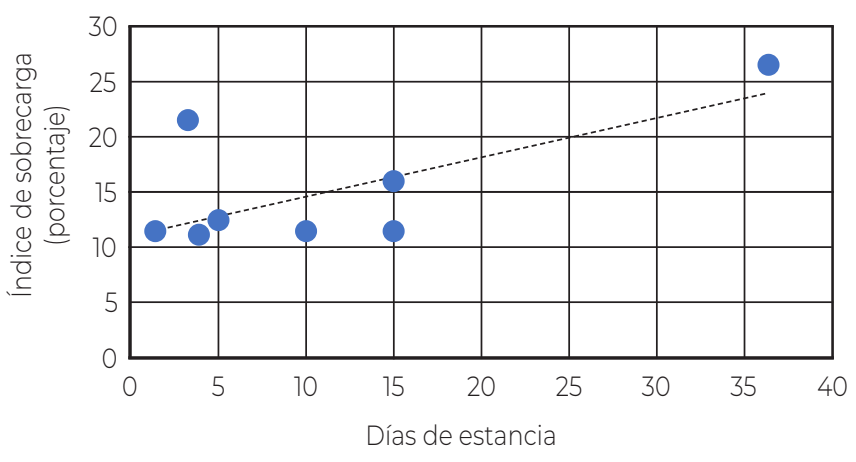

Figura 6: Relación entre días de estancia e IS > 10\% en cirugía general.

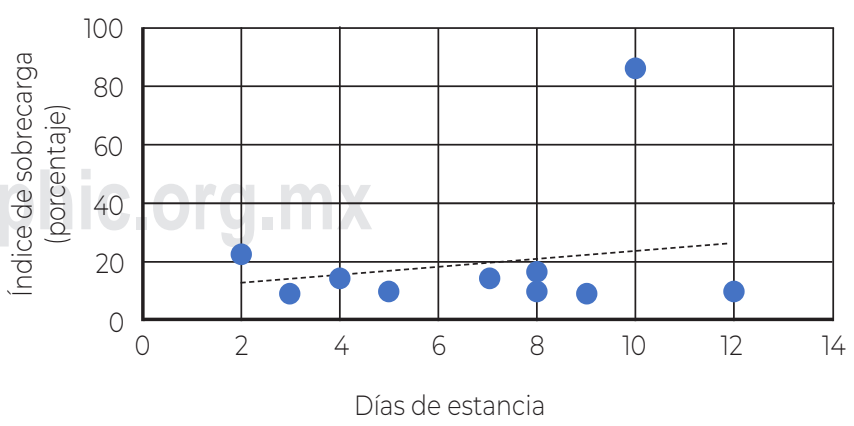

Figura 7: Relación entre días de estancia e IS > 10\% en Medicina Interna. 


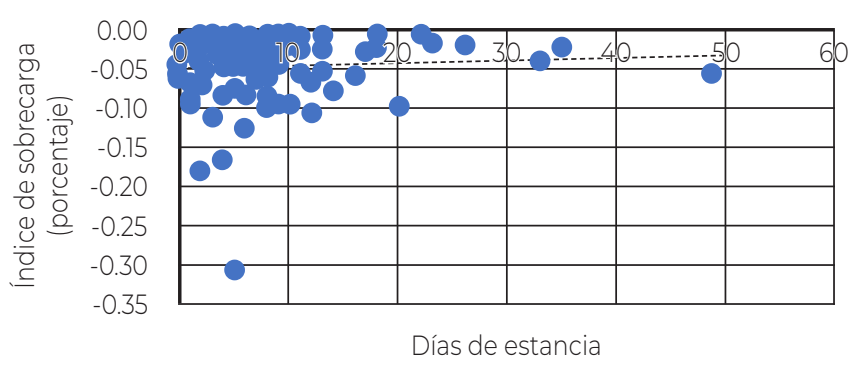

Figura 8: Relación entre días de estancia e IS negativo.

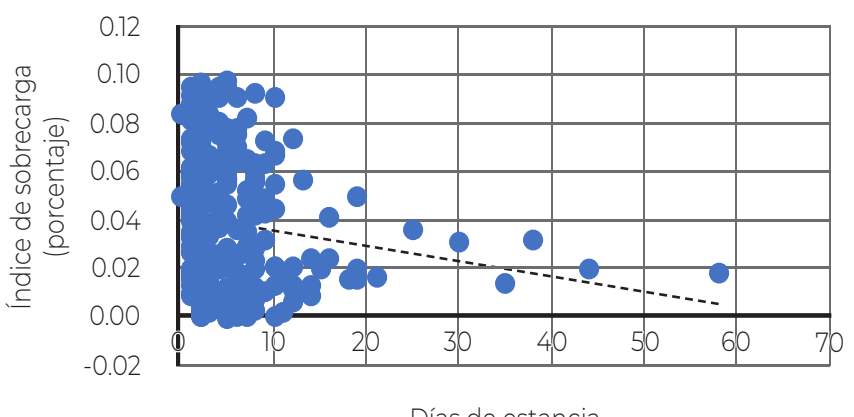

Figura 9: Relación entre días de estancia e IS de 0-10\%.

sobrecarga positivo, pero no mayor a 10\%, encontrando que en este grupo sí se observó correlación y diferencia estadística significativa $(r=0.16 ; p=$ 0.01) (Figura 9).

Debido a lo anterior, se dividió a estos pacientes en dos grupos, el primero con índice de sobrecarga mayor o igual a 0 pero menor a $5 \%$ y el segundo mayor o igual a 5 pero menor o igual que 10\%. En el grupo de 0-5\% se encontraron 161 registros, de los cuales no hay correlación o diferencia estadística significativa (Figura 10) ( $r=-0.01 ; p=0.86)$. En el grupo de 5-10\%, de 73 pacientes, tampoco se encontró correlación o diferencia estadística ( $r \leq 0.14 ; p=0.21$ ) (Figura 71). De igual forma, se realizó el análisis de varianza entre el índice de sobrecarga, el destino y el servicio para establecer la influencia del IS > 10\% en el destino del paciente en cada servicio. Se encontró que existe diferencia significativa entre destinos $\left(F_{[3 ; 386]}=4.26 ; p<0.05\right)$; sin embargo, la prueba de contrastes no destacó destino en particular (Fisher LSD Alpha: = 0.05 LSD: = 0.06) (Figura 71).

Para el caso de cada servicio, se detectaron diferencias significativas en la varianza $\left(F_{[5 ; 386]}=3.01\right.$; $\mathrm{p}<0.05)$, en la prueba de contrastes se encontró que el Servicio de Urgencias se distingue por su alta media de IS > 10\% respecto de los demás servicios (Figura 12).

\section{DISCUSIÓN}

Hasta el momento no se encontraron estudios que evaluaran la prevalencia global de sobrecarga de fluidos en pacientes hospitalizados. Diversos análisis se han realizado en poblaciones distintas, un estudio publicado en 2015 reportó una prevalencia de $86 \%$ en pacientes sobrevivientes de choque séptico. ${ }^{4}$ En otro estudio longitudinal con 405 pacientes se encontró que $67 \%$ de ellos contaban con sobrecarga de fluidos al ingreso y que éste persistía a los tres días en $48 \%$ del total. ${ }^{8}$ Si bien es cierto que los pacientes incluidos por servicio en este estudio son muy pocos en algunos casos, las prevalencias reportadas superan por mucho las encontradas en esta investigación, incluso para los servicios que atienden a pacientes críticos en el hospital de segundo nivel de México.

El balance de fluidos positivo se ha relacionado con diversas complicaciones, mortalidad, morbilidad y duración de hospitalización. Respecto de la última variable, se realizó una correlación entre los diferentes valores de sobrecarga y los días de estancia intrahospitalaria, en términos generales

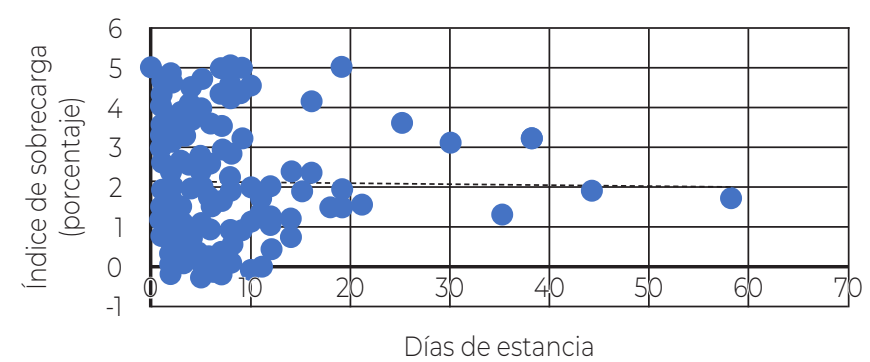

Figura 10: Relación entre días de estancia e IS de 0-5\%

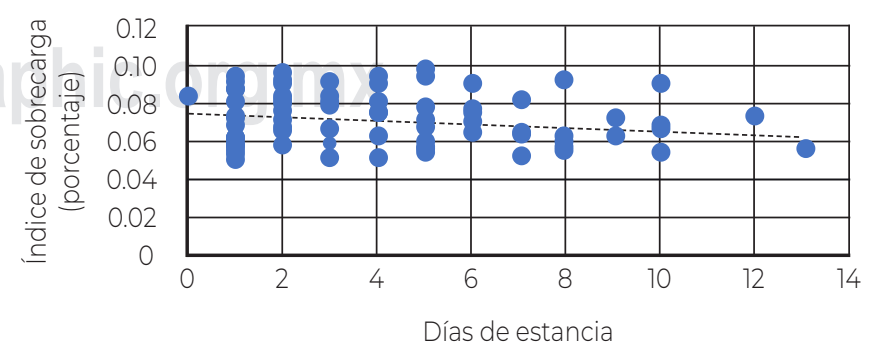

Figura 11: Relación entre días de estancia e IS de 5-10\%. 


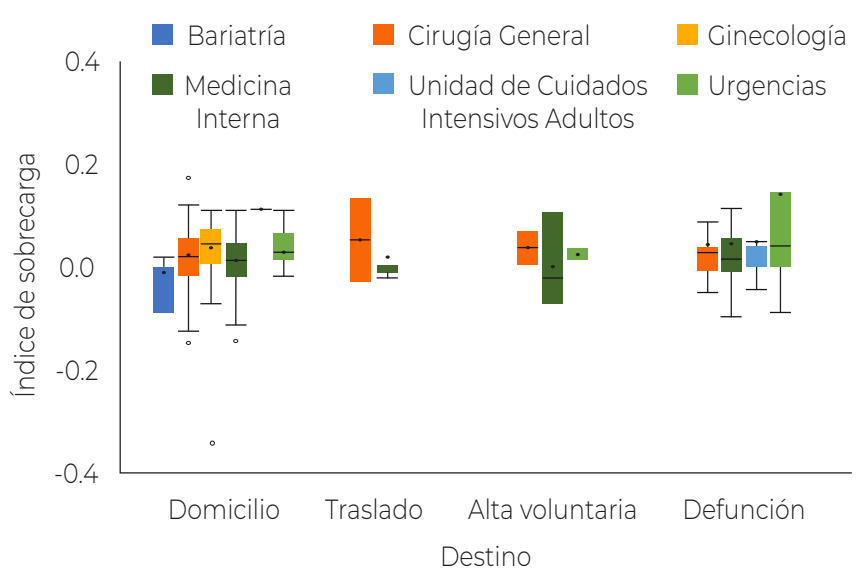

Figura 12: Diagrama de cajas, sobrecarga, destino y servicios.

no se encontró asociación estadísticamente significativa entre la sobrecarga de fluidos $>10 \%$ y la estancia. En otro estudio retrospectivo con 63,974 pacientes se encontró aumento de hasta 3.5 días de hospitalización, aumento de días de uso de ventilador mecánico y mayor tasa de readmisión en pacientes con sobrecarga hídrica. ${ }^{9}$

Hasta el momento, no se encontraron estudios que evalúen la asociación del IS > 10\% con el destino del paciente; sin embargo, existe evidencia acerca de que la sobrecarga hídrica aumenta la mortalidad en pacientes críticos con lesión renal aguda con o sin terapia de reemplazo renal. ${ }^{10,11}$ Respecto a la sobrecarga en cada servicio, se encontró que el Servicio de Urgencias tiene los índices más altos de sobrecarga de todo el hospital de segundo nivel, esto se podría estimar de acuerdo con las características de los pacientes que acuden a urgencias, puesto que se encuentran con un estado de gravedad mayor (politraumatizados, pacientes con infarto agudo al miocardio, con evento vascular cerebral, nefropatía).

\section{CONCLUSIONES}

De acuerdo con los resultados obtenidos, se logró enfatizar que el personal de enfermería y equipo de salud que intervienen en el manejo de fluidos electrolíticos en el paciente hospitalizado es de gran importancia para su disminución de estancia hospitalaria y egreso, debido a que es fundamental para evitar complicaciones derivadas de la sobrecarga de fluidos en las que predominan fallas orgánicas, además para brindar un correcto manejo de terapía hídrica durante su estancia, y por consiguiente como un efecto secundario disminuir costos al sistema de salud pública.

\section{AGRADECIMIENTOS}

A la institución que participó en este proyecto, ya que sin el apoyo de la misma no hubiera sido posible llevar a cabo dicha investigación. A la participación indiscutible de las personas con su alto grado de experiencia laboral y académica, que ha sido parte de este proceso durante estos meses.

\section{BIBLIOGRAFÍA}

1. Henríquez-Palop F, Antón-Pérez G, Marrero-Robayna S, Conzález-Cabrera F, Rodríguez-Pérez JC. Water overload as a biomarker for heart failure and acute renal failure. Nefrologia [Internet]. 2013; 33 (2): 256-265. Disponible en: http://www.ncbi.n/m.nih.gov/pubmed/23364578

2. National Institute for Heath and Care Excellence. Intravenous fluid therapy in adults in hospital Guidance and guidelines NICE [Internet]. 2013. Disponible en: https://www.nice.org.uk/guidance/cg174/chapter/7Recommendations\#resuscitation-2

3. Hung S, Kuo K, Peng C, Wu C, Lien Y, Wang Y et al. Volume overload correlates with cardiovascular risk factors in patients with chronic kidney disease. Kidney Int. 2013; 85 (3): 703-709.

4. Mitchell KH, Carlbom D, Caldwell E, Leary PJ, Himmelfarb J, Hough CL. Volume overload: prevalence, risk factors, and functional outcome in survivors of septic shock, Ann Am Thorac Soc. 2015; 12 (12): 1837-1844.

5. Naveda OE, Naveda AF. Balance hídrico positivo y alta mortalidad en niños con sepsis grave y choque séptico. Pediatría. 2016; 49 (3): 71-77. Disponible en: https://doi. org/10.1016/j.rcpe.2016.06.001

6. Bouchard JE, Mehta RL. Fluid balance issues in the critically ill patient. Contrib Nephrol. 2010; 164: 69-78. doi: 10.1159/000313722.

7. Andrzejewski C Jr, Casey MA, Popovsky MA. How we view and approach transfusion-associated circulatory overload: pathogenesis, diagnosis, management, mitigation, and prevention. Transfusion. 2013; 53 (12): 3037-3047. doi: 10.111// trf.12454.

8. Kelm DJ, Perrin JT, Cartin-Ceba R, Gajic O, Schenck $L$, Kennedy CC. Fluid overload in patients with severe sepsis and septic shock treated with early goaldirected therapy is associated with increased acute need for fluid-related medical interventions and hospital death. Shock. 2015; 43 (1): 68-73. doi: 10.1097/ SHK.0000000000000268. 
9. Child DL, Cao Z, Seiberlich LE, Brown H, Greenberg J, Swanson A et al. The costs of fluid overload in the adult intensive care unit: is a small-volume infusion model a proactive solution? Clinicoecon Outcomes Res. 2014; 7 : 1-8. doi: 10.2147/CEOR.S72776.

10. Koonrangsesomboon W, Khwannimit B. Impact of positive fluid balance on mortality and length of stay in septic shock patients. Indian J Crit Care Med. 2015; 19 (12): 708-713.

11. Vaara ST, Korhonen AM, Kaukonen KM et al. Fluid overload is associated with an increased risk for 90-day mortality in critically ill patients with renal replacement therapy: data from the prospective FINNAKI study. Crit Care. 2012; 16 (5): R197. 